\title{
AC 2011-1443: EFFECTS OF AN INTEGRATED CALCULUS REFORM PROJECT FOR ENGINEERING MAJORS
}

\section{Po-Hung Liu, National Chin-Yi University of Technology}

Po-Hung Liu is a Professor of College of General Education at National Chin-Yi University of Technology in Taiwan. His research interests are AMT (advanced mathematical thinking) and HPM (history and pedagogy of mathematics).

Ching Ching Lin, National Taipei University of Technology

Tung-Shyan Chen, Fundamental General Education Center, National Chin-Yi University of

Tung-Shyan Chen is an associate professor of the Fundamental General Education Center at the National Chin-Yi University of Technology, Taichung, Taiwan since 2010. Dr. Chen received his PhD (2002) from the Department of Mathematics at National Cheng Kung University, Taiwan.

\section{Chiu-Hsiung Liao, Fundamental General Education Center, National Chin-Yi University of Technology}

Chiu-Hsiung Liao received BS in Mathematics at National Kao-Hsiung Normal University in 1971 and MS in Mathematics at Pittsburg State University, Kansas, United States, in 1997. He has joined the faculty of National Chin-Yi University of Technology, Taichung, Taiwan, since 1981. Currently, he is an associate professor of Fundamental General Education Center at National Chin-Yi University of Technology.

\section{Yen Tung Chung, Fundamental General Education Center, National Chin-Yi University of Technology}

Yen-Tung Chung received MS in Applied Mathematics at Tsing-Hwa University in 1981. He is an assistant professor in Fundamental General Education Center, National Chin-Yi University of Technology.

P. C. Lin, Fundamental General Education Center of National Chin-Yi University of Technology, Taiwan R.O.C.

Ruey-Maw Chen, National Chinyi University of Technology

Ruey-Maw Chen, he was born at Tainan, Taiwan, R.O.C. He received the B. S., the M. S. and the PhD degree in engineering science from National Cheng Kung University of Taiwan R.O.C. in 1983, 1985 and 2000, respectively. From 1985 to 1994 he was a senior engineer on avionics system design at Chung Shan Institute of Science and Technology (CSIST). Since 1994, he is a technical staff at Chinyi Institute of Technology. Since 2002, he has been with the Department of Computer Science and Information Engineering, National Chinyi University of Technology (NCUT), where he is an assistant professor. His research interests include meta-heuristics, optimization and computer networks. 


\section{Effects of an Integrated Calculus Reform Project for Engineering Majors}

\section{Introduction}

It has been over two decades since the Tulane Conference was held in 1986, the birthplace of calculus reform. The appeal made by the conference-Toward a Lean and Lively

Calculus ${ }^{[4]}$ _not only initiated the calculus reform movement in the United States, but for the first time ever motivated numerous research mathematicians to engage in curriculum development. Despite reports of several promising empirical findings, a widely held conclusion of the effect of calculus reform is still in debate. The equivocal consequence is due to the universal goal of teaching calculus being unattainable and a standard evaluation method that is lacking. Calculus curriculum came under scrutiny for several reasons. First, traditional training in calculus, stressing rote calculating and practice, hinders students from gaining a higher level of conceptual understanding and fails them in studying advanced mathematics courses. Second, somewhat related to the first reason, a high failure rate in calculus forces college students to leave engineering or keeps them from choosing a mathematics-related career. Third, the faculty outside mathematics usually complains that students are ill-prepared to apply learned skills and concepts to solve practical problems. Reform effort in calculus curriculum aims to restructure content and develop tools to fix aforementioned pessimistic situations. We will briefly review calculus reform projects, then follow with a report on the PLEASE project.

\section{A brief review of calculus reform}

Rooted in its rigorous development in history, traditional instruction in calculus is conducted in logical order in which proving theorems and propositions deductively, based upon definitions and lemmas, plays a critical role; and working exercises with paper and pencil become the dominating mode of learning. This formal approach secures the foundation of calculus, but at the expense of students' intuitive understanding of the discipline. Some mathematicians thus made an urgent call for restructuring calculus curriculum.

Responding to the appeal of the Tulane Conference, Brown, Porta, and Uhl ${ }^{[2]}$ reduced calculus curriculum by deleting several topics, such as Roll's Theorem and Riemann sum definition of integral, and integrated technology into the curriculum instead. They claimed that certain topics contained in traditional textbooks are only to fool students into the belief that they have learned something ${ }^{[3]}$. Among all reform curricula, Harvard Calculus Consortium ${ }^{[6][7]}$ is the most widely adopted text and has received the greatest attention. Harvard Calculus Consortium reflected the reformed idea of "The Rule of Three" declaring that every topic should be presented in geometrical, numerical, and algebraic ways. Furthermore, it de-emphasized deductive symbolic reasoning by decreasing some sections, and stressed students' ability of application by connecting formal definitions and procedures with practical problems. For instance, Calculus: Early Transcendentals ${ }^{[13]}$, one of the 
best-selling textbooks and regarded as the most traditional textbook at that time, used five sections for discussing the concept of limit, whereas there was only one section on limits in the Harvard Calculus Consortium.

Johnson $^{[8]}$ reported on the effect of the Harvard Calculus Consortium at Oklahoma State University by comparing students' performance in reformed and traditional calculus. He indicated that reformed classes outperformed traditional classes in Calculus I and II. However, traditional Calculus I students' subsequent performance in mathematics-related courses were better than their counterparts in reformed Calculus I classes. Furthermore, $44 \%$ of reformed Calculus I students changed to traditional Calculus II programs and only $18 \%$ of traditional Calculus I students shifted to reformed Calculus II. Baxter, Majumdar, and Smith ${ }^{[1]}$ also surveyed reformed and traditional calculus students' achievement in the Math-ACT and found that traditional Calculus I students' average grade was slightly higher than that in the reformed Calculus I, but only $52 \%$ of traditional Calculus I students passed the exam, significantly lower than reformed Calculus I students' passing rate of 64\%. As for succeeding performance, reformed Calculus I students surpassed the traditional students in Physics I and Calculus II, yet traditional Calculus I students did better in Physics II and Calculus III. These outcomes seemingly suggest that the effect of the reformed curriculum may decline as the difficulty of the content increases. It appears that the experimental consequences of the reformed curriculum are hard to summarize in a single sentence. Silverberg ${ }^{[12]}$ proposed that the reformed curriculum may be more effective for those with a weak mathematics background.

\section{Critics and influence of calculus reform}

Despite its success in several aspects of helping students to master calculus concepts, some professional mathematicians remain doubtful as to what has really been achieved by reform movements. While responding to Mumford's (1997) $\operatorname{arguments}^{[11]}$, Klein and Rosen ${ }^{[9]}$ condemned reform supporters by saying that they create a straw man - the traditional calculus curriculum - and blame all faults on it. Reformers put forth various solutions, such as eliminating theories and increasing use of computers, without any scientific evidence. What if they were wrong in identifying the cause of students' failure? In their eyes, traditional calculus actually gives students the opportunity to have a deeper understanding of the subject and reform texts hinder motivated students from developing advanced thinking. Klein and Rosen satirized that calculus reform movements are not for the millions but \$millions.

Feffer and Petechuk ${ }^{[5]}$ took a neutral stance. They agreed that reform curriculum may help students be more capable of connecting mathematics with the real world but, in their eyes, democratizing the curriculum by reducing its rigor actually is just watering it down. 
Feffer and Petechuk emphasized that calculus should not be expected to apologize for being difficult because skills will help students succeed.

Though calculus reform receives praise as well as criticism, there are some signs revealing its positive influence on textbook development. Calculus: Early Transcendentals ${ }^{[13]}$ was once regarded as the representative of the traditional textbook, yet Stewart ${ }^{[14]}$ claimed in his recent edition that:

When the first edition of this book appeared eight years ago, a heated debate about calculus reform was taking place. Such issues as the use of technology, the relevance of rigor, and the role of discovery versus that of drill, were causing deep splits in mathematics departments....In this third edition, I continue to follow that path by emphasizing conceptual understanding through visual, numerical, and algebraic approaches.

The principal way in which this book differs from my more traditional calculus textbooks is that it is more streamlined....I don't prove as many theorems....([14], xiii-xiv).

It appears that, as Stewart pointed out, both reformers and traditionalists have realized that enabling students to understand and appreciate calculus is their common goal. We are convinced that any reform effort should keep track of this common goal.

\section{PLEASE project}

Taiwanese students often demonstrate high mathematics capability in international comparisons (e.g., TIMSS and PISA), yet this seemingly promising accomplishment may not guarantee their success in the learning of calculus. In Taiwan, calculus is usually taught in a traditional way that practice-makes-perfect is the chief belief and lecture followed by doing exercises is the major mode of teaching and learning. Additionally, all freshmen are required to take calculus regardless of their initial status in mathematics, contributing to a high failure rate. The National Science Council in Taiwan therefore initiated several projects in 2008 for improving teaching and learning in calculus.

The PLEASE project adopts a collaborative model consisting of four individual projects conducted by Mathematics and Engineering faculties at two technological universities in Taiwan. The title PLEASE stands for six main themes of this integrated project: (1) $\mathrm{P}$ - pre-calculus, (2) L-low achievers' learning, (3) E-e-learning, (4) A-assessment, (5) $\mathrm{S}$ - statistics and calculus, (6) E- engineering mathematics and calculus. The PLEASE project assumes a collaborative model not only for reforming calculus curriculum itself, but also for establishing an e-learning and assessment platform. It can be divided into three 
components: PEA, LEA, and SEA covering an extended range of curriculum from pre-calculus to post-calculus courses (Figure 1).

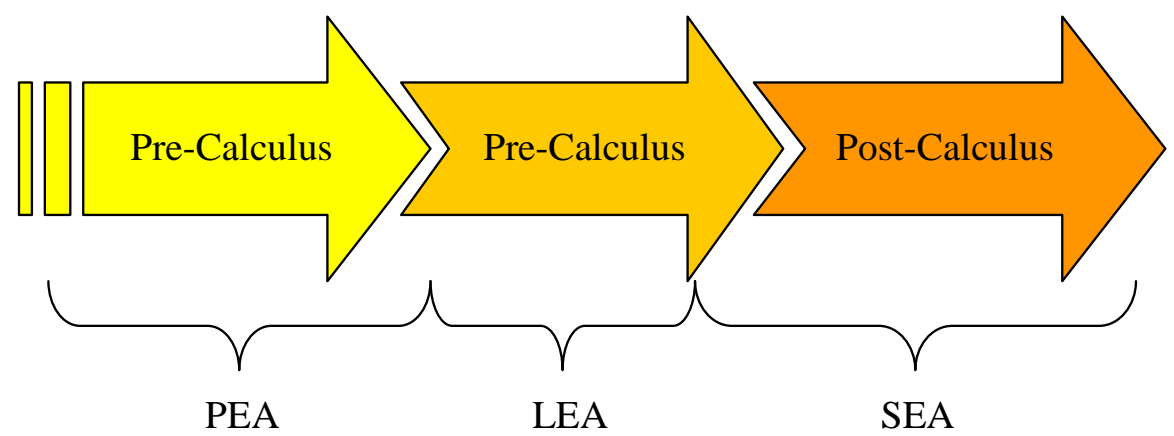

Figure 1 Relationship of the three components

PEA component: Technological universities in Taiwan mostly recruit students graduating from vocational high schools, stressing more skill training and practical knowledge. Such an instrumentalist approach may restrict college freshmen's conceptual understanding of fundamental topics in calculus, such as the concept of functions and limits. By following the Rule of Three (every topic should be presented geometrically, numerically, and algebraically), the PEA (pre-calculus, e-learning, and assessment) component combines Calcai, a graphical software, and Mimic Builder, an e-learning device, to develop an e-learning tool for pre-calculus. It enables non-technical users to create the e-learning courses by using the PowerPoint file and working with the assistant Tablet digital pen. Furthermore, a web-based test bank is established for assessing college freshmen's concept knowledge in pre-calculus and evaluating the effect of this e-learning tool.

LEA component: High failure rate in calculus is not uncommon in Taiwan's universities. Despite their outstanding performance on international assessments in mathematics, such as TIMSS and PISA, Taiwanese students' attitudes toward mathematics have been reported to be very low ${ }^{[10]}$. Poor attitudes, to a great extent, weaken the college freshmen's driving force to learn calculus, which is essential for their majors. The LEA (low achievers, e-learning, and assessment) component attempts to construct an auxiliary environment, including a computerized adaptive diagnosis evaluation system and teaching assistants, to enhance low achievers' learning. In order to identify difficulties in learning calculus, students were asked to respond to items from the web-based evaluation system. Because the computerized adaptive test (CAT) is knowledge-structured and hierarchical, we may locate their obstacles in learning calculus and develop a tailored curriculum. Moreover, selected teaching assistants were trained to help the execution of the tailored curriculum and serve as instructors outside the classroom. 
SEA component: Calculus reform is usually criticized in that it may produce deficiencies in preparing students to take advanced mathematical courses. Additionally, one of the engineering majors' major obstacles in taking post-calculus courses was how to apply learned concepts in a new context. The SEA (statistics and calculus, engineering mathematics and calculus, and assessment) component deals with this issue by restructuring calculus curriculum to help students make a connection between calculus and subsequent mathematical courses, such as statistics and engineering mathematics. Several fundamental concepts in statistics (e.g., the expected value of the function of discrete stochastic variable and continuous stochastic variable) require sophisticated understanding of infinite series and definite integral, which are difficult for engineering majors to comprehend. A particular emphasis on these topics was made to fit their future needs in studying statistics.

Similarly, engineering majors usually have trouble grasping complicated concepts and processes of engineering mathematics such as differential equations, Fourier series, and Laplace transform, all of which entail a solid background in integrals as well as in differentials. In our calculus curriculum, students were trained to construct and solve mathematical models of given realistic problems by introducing them to the solutions of basic types of differential equations, Fourier series, and Laplace transform.

\section{Results}

PEA component: A test bank consisting of 286 pre-calculus problems was created to assess freshmen's fundamental ability in mathematics. The distribution of the problems is shown in Table 1 in which almost all of the main topics of high school mathematics were included. Actually, some basic conceptions of calculus, such as differentiations and integrations of polynomials, are covered by high school mathematics, but they were not the targets of our study.

Table 1 Distribution of Problems in the Test Bank

\begin{tabular}{lc}
\hline \multicolumn{1}{c}{ Topic } & Number of problems \\
\hline number and equations & 51 \\
inequalities & 25 \\
Cartesian coordinate system & 30 \\
lines & 19 \\
conic sections & 51 \\
exponential and logarithmic functions & 40 \\
trigonometric functions & 47 \\
vectors & 23 \\
\hline
\end{tabular}

There were 1,172 first-year engineering majors participating in the study and 185 of them 
were assigned as an experimental group according to their original departments. Prior to the Fundamental Mathematics Test (FMT), the experimental group was allowed to use the Learning Assistant System (LAS) in which a window showing problem-solving procedures involving graphs and verbal explanations would pop up if the solver chose a wrong answer (Figure 2). Students were able to re-activate the whole procedure as they like, but repeat was not possible if the window was closed. Therefore, it was expected that students would attain full understanding before they turned the system off.

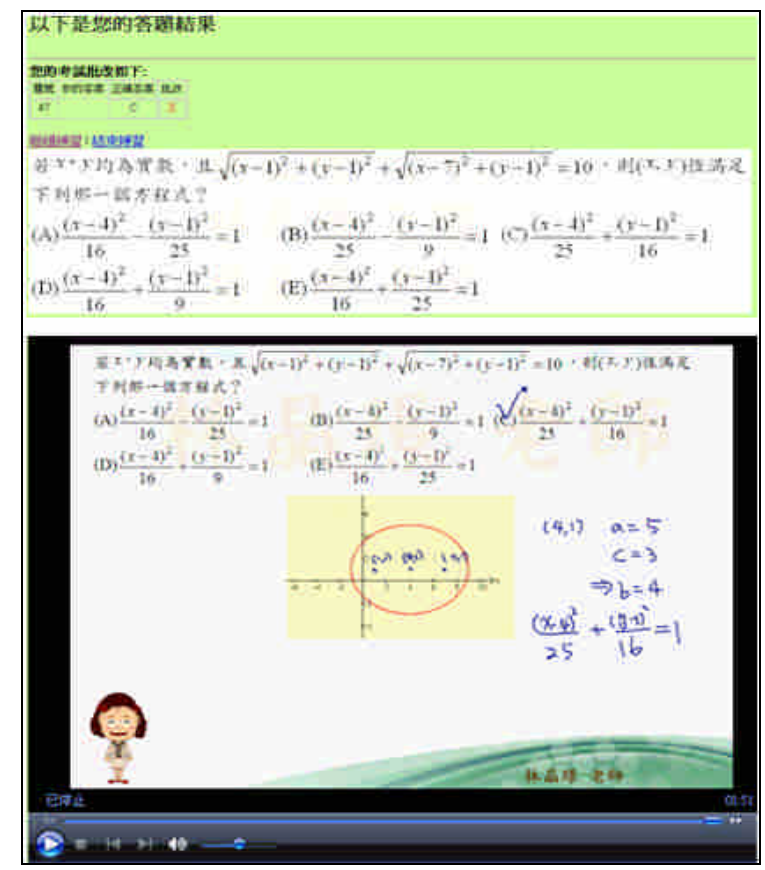

Figure 2 An example of LAS window

Table 2 Comparison of the two groups' test scores

\begin{tabular}{lcclccc}
\hline & $\mathrm{N}$ & $\begin{array}{c}\text { Mean } \\
(\mathrm{SD})\end{array}$ & $\begin{array}{l}\text { Levene Test } \\
\text { F-value }\end{array}$ & $\begin{array}{c}\text { Levene test } \\
p \text {-value }\end{array}$ & $\begin{array}{c}t \text {-test } \\
t \text {-value }\end{array}$ & $\begin{array}{c}t \text {-test } \\
p \text {-value }\end{array}$ \\
\hline Experimental Group & 185 & $\begin{array}{c}64.49 \\
(15.36)\end{array}$ & & & & \\
Control Group & 987 & $\begin{array}{c}61.06 \\
(15.55)\end{array}$ & & & & \\
\end{tabular}

${ }^{*} p<.005$

The result showed that the average of the experimental group on FMT was 64.49 and the control group was 61.06. The F-value of Levene test was 0.00 with resulting p-value 0.99 , indicating the null hypothesis of the population variances was equal, which was insignificant. 
Based upon the assumption of equal variance, the $t$-test was conducted and revealed that $t=$ 2.75 with $p$-value 0.003 , suggesting the experimental group outperformed the control group on FMT at the 0.005 significance level (Table 2).

LEA component: LEA component consists of two parts whose purpose of LEA was to improve students' learning in calculus via an interactive visualization tool (IVT) and computerized adaptive testing (CAT). The IVT in the study was developed using Java applets to encourage learners' intuitive thinking and enhance their visualization ability in calculus. On the platform of IVT, users were first shown concepts relative to each topic followed by instructions for implementing the interactive applets. Some illustrative concepts of calculus with animation or interactive Java interface then appeared. For instance, for improving students' understanding of the relationship between the secant line and tangent line, we specified any four real numbers $c_{0}, c_{1}, c_{2}, c_{3}$, to graph the polynomial function of the form

$$
f(x)=c_{0}+c_{1} x+c_{2} x^{2}+c_{3} x^{3}
$$

we then chose a real number $c$ by moving the slider, fixed the number $c$ and let $P(c, f(c))$ be the point of tangency on the graph of $f$. The tangent line (red line) to the graph $f$ at the point $P$ is shown in Figure 3. We then can approximate this slope using a secant line through the point $P$ and a second point on the graph $f$. In this IVT, as second point $Q(c+h, f(c+h))$ moves along the curve $f$ towards point $P$ by changing the value of $h$ from 1 to 0 , the secant line through points $P$ and $Q$ will approach the tangent line at point $P$ (see Figure 3 ). The slope " $m_{\text {sec }}$ " of the secant line through points $P$ and $Q$ is

$$
m_{\mathrm{sec}}=\frac{f(c+h)-f(c)}{(c+h)-c}=\frac{f(c+h)-f(c)}{h} .
$$

The specific value of the right-hand side of this equation is a difference quotient. As point $Q$ moves along the curve $f$ towards point $P$, that is, as $Q$ approaches $P$, the slope $m_{\text {sec }}$ of the secant line will approach the slope $m_{\text {tan }}$ of the tangent line. Students were allowed to implement this dynamic figure to explore how the different quotient $m_{s e c}$ approximates to the slope $m_{\text {tan }}$ by changing the value $h$ from 1 to 0 as point $Q$ moves along the curve $f$ towards point $P$. Thus 


$$
m_{\mathrm{tan}}=\lim _{h \rightarrow 0} m_{\mathrm{sec}}=\lim _{h \rightarrow 0} \frac{f(c+h)-f(c)}{h} .
$$

The whole dynamic process is revealed in Figure 3.

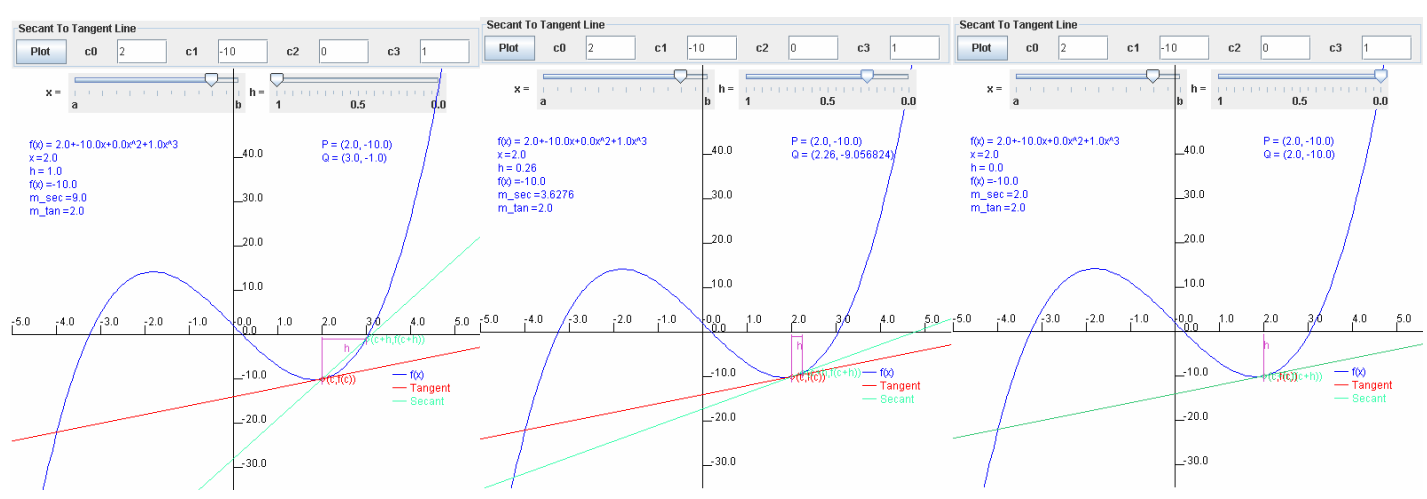

Figure 3 Tangent of the polynomial function $f(x)=2-10 x+0 x^{2}+x^{3}$ at the point $(2,-10)$.

A total of 12 college freshmen (4 females and 8 males), demonstrating difficulties in grasping differential and integral concepts, were invited to participate in the clinical investigation. To investigate participants' initial status, they were asked to answer a set of multiple-choice questions on a worksheet concerning three topics-(1) secant line and tangent line, (2) area and Riemann sum, and (3) solid of revolution, followed by interviews for detecting their misconceptions. After the pre-test, participants then partook in a computer-based clinical instruction through IVT in a computer lab. Following the clinical instruction, participants were asked to respond to the second worksheet containing equivalent but different problems with the first worksheet. The results show participants achieved higher scores on the post-test than on the pre-test (Table 3). Readers may refer to Liu et al. (manuscript in preparation) for the details of the whole process.

The second part of LEA was assessing students' abilities by means of computerized adaptive testing (CAT), a test based upon the examinee's ability levels. CAT was designed to detect examinees' potential by successively selecting appropriate tasks on the basis of their previous responses. If examinees were doing well on an easy problem with basic concepts, they would then be presented with a more difficult one. On the other hand, once they performed poorly on a difficult item requiring higher level thinking, a simpler or lower level problem would be shown to them. The advantage of CAT is that the difficulty of the exam was tailored to examinees' level of ability. A total of 120 engineering majors from two 
distinct classes participated in a pre-test consisting of problems of indefinite integral, yielding a mean score 72.09 by taking 17.15 problems on average. Low achievers then received two-weeks of remedial instruction assisted by teaching assistants followed by a post-test. Results indicate that the participants scored significantly higher than they did on the pre-test and used fewer items (10.73 on average) to finish the test (Table 3). Further inquiry revealed that the effects were also significant for two distinct classes (Table 4). The overall outcomes suggest the remedial instruction did improve students' understanding of indefinite integral and CAT was an efficient and economic tool for assessing their progress.

Table 3 Paired Sample Test for pre-test and post-test

\begin{tabular}{lccccc}
\hline & Mean & SD & $\mathrm{N}$ & $t$-value & $p$-value \\
\hline Pre-test & 72.09 & 21.22 & 120 & & \\
Post-test & 88.75 & 14.56 & 120 & 9.172 & $0.000^{*}$ \\
\hline
\end{tabular}

${ }^{*} \mathrm{P}<.005$

Table $4 \underline{t \text {-test for two classes' achievements on pre-test and post-test }}$

\begin{tabular}{lcccccc}
\hline & $\begin{array}{c}\text { Pre-test } \\
\text { Mean }\end{array}$ & $\begin{array}{c}\text { Post-test } \\
\text { Mean }\end{array}$ & Difference & SD & $t$-value & $p$-value \\
\hline Class A & 71.38 & 90.7 & 19.32 & 18.81 & 7.593 & $0.000^{*}$ \\
Class B & 72.8 & 86.8 & 14.00 & 20.74 & 5.229 & $0.000^{*}$ \\
\hline${ }^{*} p<.005$ & & & & & &
\end{tabular}

SEA component: Rather than treating calculus as an independent subject, SEA aimed to develop curricula connecting calculus with statistics and engineering mathematics via an application-based approach. The two projects are still in the data collection stages, only preliminary results are reported.

SEA stressed the application of mathematical concepts such as an improper integral problem involving probability concepts and a quiz was presented to students as shown in Table 5. The problem ( $a$ ) may be solved by finding the value of $\int_{10}^{\infty} \frac{1}{5} e^{-\frac{x}{5}} d x$ directly and Table 6 shows the experimental group did not outperform the control group. However, a higher ratio of students in the experimental group were successful with the problem $(b)$ since it required students to distinguish variables $x, t$, and $\theta$. Probably attributable to their regular training, the experimental group was able to realize the different representation for each variable and was less likely to be confused by multiple variables. 
Table 5 A quiz for SEA component

(a) Let $x$ represent the time interval (measured in minute) for calling 911 on Halloween night and assume its probability model is $f(x)=\frac{1}{5} e^{-\frac{x}{5}}, 0<x<\infty$. Find the probability $\mathrm{P}$ for the time interval greater than 10 minutes $(x>10)$.

(b) The probability density function of a variable with exponential distribution is $f(x)=\frac{1}{\theta} e^{-\frac{x}{\theta}}, 0<x<\infty, \theta>0$. Find the moment-generation function $M(t)$ of $x$ $\left(M(t)=\int_{0}^{\infty} e^{t x} \frac{1}{\theta} e^{-\frac{x}{\theta}} d x\right)$.

Table 6 Statistics of students' performance on the application problem

\begin{tabular}{llcc}
\hline & $\mathrm{N}$ & Number/Ratio of solving $(a)$ & Number/Ratio of solving $(b)$ \\
\hline Experimental Group & 52 & $44(84.6 \%)$ & $30(57.7 \%)$ \\
Control Group & 54 & $48(88.9 \%)$ & $25(46.3 \%)$ \\
\hline
\end{tabular}

The second project of SEA prepared students' preliminary mathematical background by introducing concepts of differential equation, and Laplace and Fourier Transform. For instance, convolution filter is a commonly-used image processing technique and Figure 3 demonstrates how convolution filter works. After introducing improper integral and infinite series, the lecturer presented the model and reminded students of its relationship with Laplace and Fourier Transform and their application in convolution filter. It was expected to enhance students' concept image related to the topic and increase their motivation to learn. Follow-up interviews found that students were impressed by the supplementary material though they did not actually realize how it might help their subsequent study in engineering mathematics.

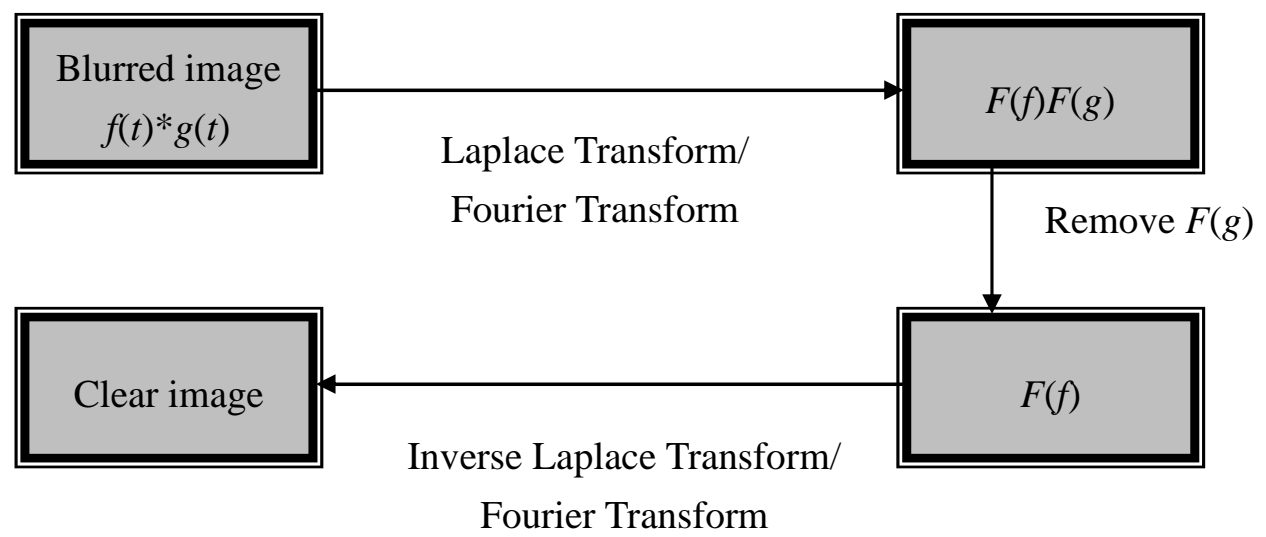

Figure 3 Process of convolution filter 


\section{Conclusion}

The present study investigated the effects of an integrated project responding to the appeal of calculus reform. As the enrollment rate continually increases, more and more Taiwanese universities find first-year engineering majors' mathematics backgrounds are not sufficient to meet the basic requirement of calculus. However, preliminary courses such as pre-calculus and college algebra that prepare students for calculus are not available in Taiwan. PEA thus offered a time-saving e-learning approach for teachers trying to make up for students' deficiencies in learning calculus. Furthermore, low achievers always fail on standardized tests and have difficulty grasping significant concepts of calculus. LEA developed CAT to measure individuals' abilities based upon their capability, and established an IVT platform to build up their intuitive understanding. Though there was little evidence indicating that this line of effort may prepare them to succeed on traditional standardized tests, LEA not only empowered teachers with an economic method for assessment, but provided students facing obstacles in calculus an alternative way of learning. The purpose of the third component SEA was to reveal the value of calculus reform. Because learning is a continuous and mutually connected process, rather than singular and isolated, it is our belief that any curriculum reform effort in engineering education would not be deemed as promising if it failed to assist students' future learning in related mathematical courses.

Note that the approach mentioned above may be controversial. In order to avoid the debates occurring in calculus reform, we set the rigor issue aside, which might be intolerable for many who insist on the tradition of mathematical culture. Students in our study were graduates from vocational high schools and were studying at technological universities, usually less theoretical in their professional training. Therefore, we were allowed to restructure and practice the whole reform curriculum. Nonetheless, we should remember that whether the whole program consisting of four individual projects can be organized into a single course is questionable. We realize and note that this will be a major challenge for upcoming endeavors.

Postscript: A list of information for the abbreviations used in the text can be referred to the Appendix.

\section{Bibliographies}

[1] Baxter, J., Majumdar, D., \& Smith, S. (1998). Subsequent grades assessment of traditional and reform calculus. PRIMUS, 8(4), 317-330.

[2] Brown, D., Porta, H. A., \& Uhl, J. J. (1990a). Calculus \& Mathematica: Courseware for the Nineties. The Mathematica Journal, 1(1), 43-50.

[3] Brown, D., Porta, H. A., \& Uhl, J. J. (1990b). University of Illinois: Calculus and Mathematica. In T. W. 
Tucker (Ed.), Priming the calculus pump: Innovations and resources (pp. 101-120). Washington, DC: The Mathematical Association of America.

[4] Douglas, R. G. (ed.) (1986). Toward a Lean and Lively Calculus. MAA Notes Number 6, Mathematical Association of America, Washington, DC.

[5] Feffer, L. B., \& Petechuk, D. (2002). Has the calculus reform project improved students' understanding of mathematics?

http://www.scienceclarified.com/dispute/Vol-2/Has-the-calculus-reform-project-improved-students-understa nding-of-mathematics.html, last accessed 20 September 2010.

[6] Hughes-Hallett, D., \& Gleason, A. M., et al. (1992). Calculus (preliminary edition), John Wiley \& Sons, New York

[7] Hughes-Hallett, D., \& Gleason, A. M., et al. (1994). Calculus (international edition), John Wiley \& Sons, New York.

[8] Johnson, K. (1995). Harvard calculus at Oklahoma State University. The American Mathematical Monthly, 102(9), 794-797.

[9] Klein, D., \& Rosen, J. (1997). Calculus reform—for the \$millions. Notices, 44(10), 1324-1325.

[10] Mullis, I.V.S., Martin, M.O., \& Foy, P. (2008). TIMSS 2007 International Mathematics Report. Chestnut Hill, MA: TIMSS \& PIRLS International Study Center, Boston College.

[11] Mumford, D. (1997). Calculus reform—-for the millions. Notices, 44(5), 559-563.

[12] Silverberg, J. (1999). Does calculus reform work? MAA Note, 49, 245-248.

[13] Stewart, J. (1999). Calculus: Early Transcendentals. Books/Cole.

[14] Stewart, J. (2006). Calculus: Concept \& Context. Books/Cole.

\section{Appendix}

A list of information about abbreviations

\begin{tabular}{|l|l|}
\hline CAT & Computerized Adaptive Test \\
\hline FMT & Fundamental Mathematics Test \\
\hline IVT & Interactive Visualization Tool \\
\hline LAS & Learning Assistant System \\
\hline LEA & Low achiever, E-learning, Assessment \\
\hline PEA & Pre-calculus, E-learning, Assessment \\
\hline PISA & Programme for International Student Assessment \\
\hline SD & Standard Deviation \\
\hline SEA & Statistics, Engineering Mathematics, Assessment \\
\hline TIMSS & Trends in International Mathematics and Science Study \\
\hline
\end{tabular}

